
PERFURMANCE OF AN ARRAY OF VERTICAL UIPOLES

ONER AN INHOMOGENEOUS GROUND SYSTEMT

N. C. Mathur* and R. J. Kingk*

Department of Electrical and Computer Engineering University of Wisconsin-Madison, Madison, WI 53706

* Now at Communications Laboratory

Department of Electrical Engineering and Computer science, University of Illinois at Chicago, Chicago, IL 60680

* Now at Electronics Department

Lawrence Livermore National Laboratory

Livermore, CA 94550

SUWWARY

The elevation radiation patterns of a stacked array of vertical electric dipoles (VEUs) over several different azimuthally symmetric inhomogeneous ground systems are studied using an integral formulation. As the ground influences the pattern of each VED differently, there is no known optimum array excitation function which can be used to achieve desired beam shaping and steering. Patterns in an array of 21 VEDs spaced $0.1 \lambda$ apart are complited and compared at HF (10 MHz) for three excitation functions: (a) conventional linear spacial phasing, (b) phasing accoroing to the complex conjugate of the field produced by each VED in the direction of steering, and (c) spacially sinusoidal excitation with constant phasing. Kesults are given for grounos consisting of homogeneous earth, a perfectly conoucting ground plane, a perfectly conducting disk on homogeneous earth and $2 \lambda$ long radial wire ground systems on welt ano poorly-conducting earth. It is found that the radiation pattern cannot be steered below about $y^{\circ}$ in elevation for any of the excitation functions or the ground systems used. For low-angle steering, conjugate excitation produces a slightly narrower beam with smaller sioelobes. Highly conaucting grounds tend to permit. steering to slightly higher elevations with narrower beams.

FFinancially supported by the US Air Force Uffice of Scientific Research Grant No. AFOSR-81-0233, The University of Wisconsin Engineering Experiment Station, the University of Wisconsin Research Committee, and the US Department of Energy under contract N-7405-ENG-48 to the Lakrence Livermore National Laboratory. 


\subsection{INTROCUCTION}

The behavior of a vertical array of antennas located over a ground system of arbitrary ele:trical characteristics has considerable practical significance. In HF communication and radar work, narrow beams are frequently produced by arrays. Narrow steerable beans are particularly importani in HF over-the-horizon radar. The design of such arrays located over inhomogeneous ground systems is difficult and the literature on this subject is scant. It is complicated by the fact that the radiation field of a dipole located over such a ground is complex, oifficult to evaluate, and also height-dependent. Thus, in effect, the problem is one of understanding the performance of an array of antennas of dissimilar radiation patterns. Here the performance of a vertical arraj of vertical electric dipoles (VEDs) over an inhomogeneous ground is consioered. The grouna properties are considered to be azimuthally symetric but radially varying in an arbitrary manner. Recently, Teng and King (1981) gave an intearal formulation of the problem using the electromagnetic compensation theorem. This formulation is used here to numericaliy evaluate the radiation pattern of a VEO located at an arbitrary height above the ground. The radiation pattern of a vertical array of 21 VEDs spaced $0.1 \lambda$ apart is studied using three different types of array excitation functions. One is the conventional equal-amplitude linear-phase excitation function suitable for arrays of identical radiators in free space or over a perfect ground. The second uses an excitation function which is the complex conjugate of the radiation field of each dipole in the direction of desired beam steering (Waldman, 1970). The thira uses a sinusoiaal amplitude excitation function 
with constant phase which is a special case of the complex conjugate excitation as will be shown later. The performance of the array for each type of excitation under different ground cuiditions is studied and observations are made on some general features of performance such as steering angle, beam size ano shape, and sidelobe structure.

\section{U FURMULATIUN}

In the past a number of approaches have been used to find the radiation field of a VED over an inhomogeneous ground (Hufford, 1952; Wait and Spies, 1970; de Jong, 1975; Rafuse and Ruze, 1975; balanis and DeCarlo, 1982). The formulation used here is oue to Teng and King (1981) which is based on the electromagnetic compensation theoren. Details of the formulation are given in the reference cited above. However, for the sake of completeness, the basic steps are briefly summarized below.

\subsection{The Integral Equation}

Figure 1 shows the geometry of the VED above the inhomogeneous ground which has a radially varying surface impedance in the region $0<p<b$. An elemental VED with current moment $I_{1} \bar{l}_{1}$ is located at $p=U$ and $z=h_{1}$. To find the magnetic field at $P_{2}$, a horizontal magnetic oipole (HW) of moment $k_{2} \bar{l}_{2}$ located at $\rho$ and $z=h_{2}$ is used as a test source. The $z=0$ plane is the surface $S$ and $z>0$ is free space. The media for $z \leq 0$ oetermines the surface impedance profile,

$$
Z_{S}^{\prime}(p)=n_{0} \Delta^{\prime}(0)
$$


where $\eta_{0}=120 \pi$, and the normalized surface impedance $\Delta^{\prime}(\rho)$ varies radially in magnitude and/or argument, with no dependence on the azimuthal coordinate $\phi$.

Let $\bar{E}_{1}, \bar{H}_{1}, \bar{E}_{2}$ and $\bar{H}_{2}$ be the electric and magnetic fields for the VEU (source 1) and the HMD (source 2) when the medium for $z<0$ is nomogeneous with constant surface impedance $Z_{s}=\pi_{0} \Delta_{1}$. Also, let $\bar{E}_{1}^{\prime}$ and $\bar{H}_{1}^{\prime}$ be the unknown fields of the VED when the surface impedance is changed to $Z_{S}^{\prime}(p)$. As the problem is TM to $\rho$ and $z$, i.e., $A_{i}=\bar{a}_{\phi} H_{\phi}$, a $\phi$-directed HMD test source is chosen to find $H_{l_{\phi}}^{\prime}$

The electromagnetic compensation theorem first given by Monteath (1951) and later generalized by Mittra (1961) can be stated as

$$
\int_{S}\left(\bar{E}_{1}^{\prime} \times \bar{H}_{2}-\bar{E}_{2} \times \bar{H}_{1}^{\prime}\right) \cdot \hat{n} d S=\int_{R_{2}}\left[\bar{J}_{2} \cdot\left(\bar{E}_{1}^{\prime}-E_{1}\right)-\bar{M}_{2} \cdot\left(\bar{H}_{1}^{\prime}-\bar{H}_{1}\right)\right] d v
$$

where the volume $R_{2}$ includes only source 2 and $\hat{n}$ is a unit vector nomial to $S$. We invoke the surface impedance boundary conditions,

$$
\hat{n} \times \hat{E}_{1}=-\pi_{0} \Delta_{1} H_{l_{\phi}} \dot{a}_{\phi} \text { ano } \hat{n} \times \hat{E}_{1}^{\prime}=-\eta_{0} \Delta^{\prime}(\rho) H_{l_{\phi}} \hat{a}_{l_{\phi}}
$$

in the unperturbed and perturbed cases respectively. In the present problem, $\bar{M}_{2}=K_{2} l_{2} \delta\left(P-P_{2}\right) \vec{a}_{l_{\phi}}$ and $\bar{J}_{2}=0$. Then, substituting (3) into (2) gives the basic working equation

$$
\begin{aligned}
& H_{1_{\phi}}^{\prime}\left(\rho, h_{1}, h_{2}, \Delta^{\prime}\right)=H_{1 \phi}\left(\rho, h_{1}, h_{2}, \Delta_{1}\right)+ \\
& +\frac{\eta_{0}}{K_{2} l_{2}} \int_{S}\left(\Delta^{\prime}\left(\rho_{1}\right)-\Delta_{1}\right) H_{1_{\phi}}^{\prime}\left(\rho_{1}, h_{1}, 0, \Delta^{\prime}\right)\left[\bar{a}_{1 \phi} \cdot \bar{H}_{2}\right] \Delta S .
\end{aligned}
$$


The general procedure for finding the radiation pattern using (4) is to first find the unknown field $H_{\phi}^{\prime}\left(\rho, h_{1}, 0, \Delta^{\prime}\right)$ on $S$. This is accomplished by setting $P_{2}$ on $S$, and performing a stationary phase integration to reduce the resulting integral equation to one dimension. This yields a Volterra integral equation of the second kina which is solved for $H_{i_{\phi}}^{\prime}\left(p, h_{1}, 0, \Delta^{\prime}\right)$. This result isthen inserted back into (4) which is numerically integrated in two oimensions to find the radiation field $H_{i_{\phi}}^{\prime}\left(\rho, h_{1}, h_{2}, \Delta^{\prime}\right)$ far from the VEU. The details of thisprocedure are summarized in the following. The unknown field $H_{1_{\phi}}$ is cast into the form

$$
H_{i_{\phi}}\left(\rho, h_{1}, h_{2}, \Delta^{\prime}\right)=H_{1 \phi}\left(\rho, h_{1}, h_{2}, \Delta_{1}\right) F^{\prime}\left(\rho, h_{1}, h_{2}, \Delta^{\prime}\right)
$$

where $F^{\prime}$ is an attenuation function in excess of that for propagation over $\Delta_{j}$. Substituting (5) into (4) and using only the radiation terms for the fields $H_{1 \phi}$ and $H_{2}$ (e.g., see King, 1969) yields

$$
\begin{aligned}
& F^{\prime}\left(\rho, h_{1}, h_{2}, \Delta^{\prime}\right)=1+\left[\frac{j k}{2 \pi H_{l_{\phi}}\left(\rho, h_{1}, h_{2}, \Delta_{1}\right)}\right]{ }_{S}\left(\Delta^{\prime}\left(\rho_{1}\right)-\Delta_{1}\right) \frac{e^{-j k r_{2}}}{r_{2}} . \\
& {\left[\frac{\sin \left(\phi_{2}\right) \cos (\alpha)}{\sin (\psi)+\Delta_{1}}\right\rfloor\left[\sin \left(\psi_{2}\right)+\Delta_{1} F\left(\rho_{2}, h_{2}, 0_{1} \Delta_{1}\right)\right] H_{1 \phi}\left(\rho_{1}, h_{1}, 0, \Delta_{1}\right) F^{\prime}\left(\rho_{1}, h_{1}, 0, \Delta^{\prime}\right) \Delta S,} \\
& \text { where } \\
& r_{2}=\left(\rho_{2}^{2}+h_{2}^{2}\right)^{1 / 2}, \rho_{2}=\left[\rho^{2}+\rho_{1}^{2}-2 \rho \rho_{1} \cos \left(\phi_{1}\right)\right]^{1 / 2}, \cos (\alpha)=\left(\rho_{1}^{2}+\rho_{2}^{2}-\rho^{2}\right) /\left(2 \rho_{1} \rho_{2}\right),
\end{aligned}
$$

$\sin \left(\Psi_{2}\right)=n_{2} / r_{2}, \sin \left(\phi_{2}\right)=\left[\rho_{0} \rho_{1} \cos \left(\phi_{1}\right)\right] / \rho_{2}$ ano the suppressed time factor is $e^{j \omega t}$. F is the Sommerfeld attenuation function and $\phi_{1}$ is the angle 
between the $x$-axis and $\overline{O Q}\left(=\rho_{\eta}\right)$, where $Q$ is the integration point located on $S$. To solve (6), first put the HMO on the perturbed surface $S$ by setting $h_{2}=0$. Then, (6) becomes a two-dimensional Fredholm integra] equation of the second kind where the integration extends over the perturbed portion of $S$, i.e., where $\Delta^{\prime}$ differs significantly from $\Delta_{1}$. A stationaryphase integration of the two-dimensional surface integration for $F^{\prime}\left[\rho, h_{1}\right.$, $\left.0, \Delta^{\prime}\right)$ can be reduced to a one-dimensional Volterra integral equation of the second kind (Teng and king, 1981),

$$
F^{\prime}\left(\rho, h_{1}, 0, \Delta^{\prime}\right)=1-\int_{0}^{0} F^{\prime}\left(t, h_{1}, 0, \Delta^{\prime}\right) g(\rho, t) \phi(\rho, t) d t
$$

where $\phi(\rho, t)=[t(\rho-t)]^{1 / 2}$ and

$$
\begin{aligned}
& g(\rho, t)=\left(\frac{j k}{\pi}\right)^{1 / 2} \frac{R^{2}}{\rho^{3 / 2}} \frac{\left(\Delta^{\prime}(t)-\Delta_{1}\right) t^{5 / 2}}{\left(h_{1}^{2}+t^{2}\right)\left(t+\left(h_{1}^{2}+t^{2}\right)^{T / 2}\right] / 2} F\left(0-t, 0,0, \Delta_{1}\right) . \\
& \frac{h_{1}+\left(h_{1}^{2}+t^{2}\right)^{l / 2} \Delta_{1} F\left(t, h_{1}, 0, \Delta_{1}\right)}{h_{1}+R \Delta_{1} F\left(\rho, h_{1}, 0, \Delta_{1}\right)} \cdot \frac{h_{1}+R \Delta_{1}}{h_{1}+\left(h_{1}^{2}+t^{2}\right)^{1 / 2} \Delta_{1}} e^{j k\left\lfloor R-\rho+t-\left(h_{1}^{2}+t^{2}\right)^{l / 2} j\right.}
\end{aligned}
$$

$$
\begin{aligned}
\text { Here, } K= & \left(p^{2}+h_{1}^{2}\right)^{1 / 2} \text { and } F\left(0,0,0, \Delta_{1}\right)=F(0,0,0,0)=F(t, 0,0,0)= \\
& F(p-t, U, U, 0) \equiv 1 .
\end{aligned}
$$

To obtain an approximate numerical solution of (7), the technique of product integration is used, e.g., the generalized trapezoival rule (Linz, 1969). Standard methods are not applicable due to the weak singularities $t^{-1 / 2}$ and $(p-t)^{-1 / 2}$ contained in $\phi(\rho, t)$. 
Finally, the solution for $F^{\prime}\left(0, n_{l}, 0, \Delta^{\prime}\right)$ is inserted back into $(0)$ to give $F^{\prime}\left(\rho, h_{1}, h_{2}, \Delta^{\prime}\right)$ from which the radiation pattern can be found using (5) in (4). Note that the two-dimensional integration for $F^{\prime}\left(0, h_{1}, h_{2}, \Delta^{\prime}\right)$ in $(6)$ cannot be simplified with the stationary phase method unless $\left(h_{1}+h_{2}\right) \ll p$. Therefore, Pierce's method (1957) over a planar annulus is used to perform a two-dimensional numerical integration of (6) over a perturbed circular region. Details of the numerical integration procedures using the product method and Fierce's method can be found in Teng (1978) and Teng and king (1981).

\subsection{The Surface Impedance}

This formulation requires a knowledge of the normalized surface impedance of the ground. In addition to a uniform earth, the performance of arrays over a radial wire ground system is considered. The surface impedance of such a ground system is found by considering an elemental area to be locally equivalent to a parallel wire grid of spacing $d=2 \pi \mathrm{p} / \mathrm{N}$, where $N$ is the number of radial wires and $\rho$ is the distance from the center of the ground system. The method developed by wait (1962) for finding the surface impedance of a parallel wire grid over a homogeneous ground has been used. The details are given in Teng (1978). The combined effective surface impedance $Z^{\prime}(p)$ is the parallel combination of $Z_{\text {grid }}$, the surface impedance of the parallel wire grid and $z_{\text {ground }}$, the surface impeaance of the hamogeneous ground on which the grid is placed. Thus

$$
z^{\prime}(0)=\left(z_{\text {grid }} \cdot z_{\text {ground }}\right) /\left(z_{\text {grid }}+z_{\text {ground }}\right)
$$


where $Z_{\text {ground }}=\eta_{0} \frac{k}{k^{\prime}} C^{\prime}$

and $i_{\text {grid }}=j \frac{2 \pi \rho}{N}\left\{\frac{\eta_{0}}{\lambda}\left[f_{0} \ell_{n}\left(\frac{p}{N r_{a}}\right)+\Delta f j+z_{j}\right\}\right.$

The various symbols are defined as follows:

$$
\begin{aligned}
& f_{u}=\frac{2 c^{2}\left(c^{\prime}\right)^{2}}{c^{2}+\left(c^{\prime}\right)^{2}-\left(\frac{s}{k^{\prime}}\right)^{2} \frac{L\left(k^{\prime} C^{\prime}\right)^{2}-(k C)^{2} L^{2}}{\left(k^{\prime} c^{\prime}\right)^{2}+(k C)^{2}}} \\
& \Delta f=\sum_{m=1}^{\infty}\left(g_{m}-\frac{f_{0}}{m}\right)
\end{aligned}
$$

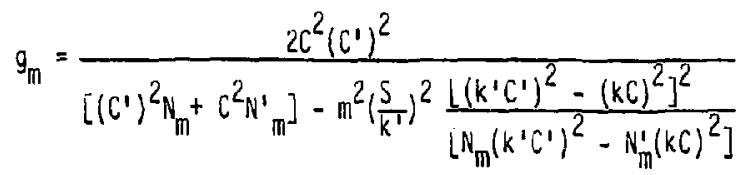

$$
\begin{aligned}
& S=\sin \theta, C=\cos \theta \\
& k=\omega\left(\mu_{0} \varepsilon_{0}\right)^{1 / 2}, k^{\prime}=\omega\left[\mu_{0}\left(\varepsilon^{\prime}-j \frac{\sigma^{\prime}}{\omega}\right)\right]^{1 / 2} \\
& k^{\prime} S^{\prime}=k S, \quad C^{\prime}=\left[1-\left(S^{\prime}\right)^{2}\right]^{1 / 2} \\
& N_{m}=\left[m^{2}-\left(k C \frac{\rho}{N}\right)^{2}\right]^{1 / 2} \\
& N_{m}^{\prime}=\left[m^{2}-\left(k^{\prime} c^{\prime} \frac{\rho}{N}\right)^{2}\right]^{1 / 2} \\
& \eta_{1}=\left[j \omega \mu_{1} /\left(\sigma_{1}+j \omega \varepsilon_{l}\right)\right]^{1 / 2}, \quad \gamma=\left[j \omega \mu_{1}\left(\sigma_{1}+j \omega \varepsilon_{l}\right)\right]^{1 / 2}
\end{aligned}
$$




$$
z_{i}=\frac{\eta_{i}}{2 \pi r_{a}} \frac{I_{0}\left(r_{a}\right)}{I_{l}\left(r_{a}\right)}=\text { Internal impedance of the wires }
$$

$I_{0}$ and $I_{1}$ are the modified Bessel functions of the first $k$ ind and of order zero and one, respectively. The first 30 terms are adequate for the summation in $\Delta f$. The radius of each wire is $r_{a}$ and $\theta$ is the angle of incidence. The earth has electrical parameters $\mu_{0}, \varepsilon^{\prime}$, and $\sigma^{\prime}$ and the wire has parameters $\mu_{1}, \varepsilon_{1}$ and $\sigma_{1}$. For sufficientiy high frequency such that $\left|\gamma r_{a}\right| \gg 1$, we can approximate $Z_{i}$ as

$$
z_{i} \simeq\left(\frac{\omega \mu}{2 \sigma_{1}}\right)^{1 / 2}\left(\frac{1+j}{2 \pi r_{a}}\right)
$$

It is assumed that $r_{a} \ll d,|k c d|<1$ and $\left|k^{\prime} c^{\prime} d\right|<1$. The amplitude and phase of $\Delta^{\prime}$ change with the angle of incidence $\theta$ but the variation is small. With the help of thes = formulas the variation of $\left|\Delta^{\prime}\right|$ with $\rho$ under two different ground conditions is shown in Fig. 2. The radial wire ground system chosen throughout this paper has $N=120$ wires of length $b=2 \lambda$ using B\&S $\# 6$ copper wires $\left(r_{a}=0.08\right\}$ in., $\left.\sigma=5.8 \times 10^{-7} \mathrm{~S} / \mathrm{m}\right)$. An operating frequency of $f=10 \mathrm{MHz}$ is assumed.

\subsection{DIPOLE RAOIATION PATTERNS}

The influence of height on the radiation pattern of the VED is studied using the above formulation. Due to the finite conductivity of the ground, the radiated field $H_{i} i_{\phi}$ is complex and both the amplitude and phase change with the height of the VED. This behavior is snown in Figs. 3 and 4 . Five different ground situations are considered, namely, (a) a radial wire 
ground system on a well-conducting earth $\left(\varepsilon_{r}=15, \sigma=10^{-2} \mathrm{~S} / \mathrm{m}\right),(\mathrm{b}) \mathrm{a}$ similar ground system on a poorly-conducting earth $\left(\varepsilon_{r}=5, \sigma=10^{-4} \mathrm{~s} / \mathrm{m}\right)$, (c) a perfectly-conducting disc of radius $2 \lambda$ over poorly-conducting ground as in (b) above, (d) a homugeneous poorly-conducting ground as in (b) with no ground system, and, (e) a perfectly conducting plane. In general, raising the height of the dipole results in the appearance of an increased number of sidelobes while at the same time the main beam becomes narrower ana gets closer to the ground. The phase increases continuously with elevation angle for dipole heights greater than U.b $\lambda$. As the dipole height is increased, the phase change with elevation angle becomes larger until at a height of $h_{1}=2 \lambda$ the phase changes through almost two complete cycles. This continuous change of phase is a direct consequence of the finite conductivity of the ground system. Radiation fielos of aipoles in free space or over perfectly conducting ground do not show a continuous phase change with elevation angle. The phase is either $0^{\circ}$ or $180^{\circ}$. The phase behavior over dissipative ground is the principal difficulty in the choice of an appropriate excitation function for an array of oipoles over such a ground. A study of Fig. 4 shows that no unique phasing function for all types of grounds can be chosen for an array of dipoles which will guarantee beam steering in a particular direction.

\subsection{ARRAY RAOIATION PATTERNS}

We now study the behavior of a vertical array of VEDs over a dissipative inhomogeneous ground. For this study we have considered an array of 2? dipoles uniformly spaced $0.1 \lambda$ apart with a total array height of $2 \lambda$. 
The problem of choosing an appropriate excitation function for beam steering for such an array has not received much attention in the literature. FitzGerell et al. (1967) have considered a vertical array of norizontally polarized antennas and have demonstrated the usefulness of a sinusoidal excitation function to achieve low-angle steering. The complex conjugate excitation function excites each element with a current which is the complex conjugate of the field produced by that element in the aesired beam direction. In the case of a perfectly conducting ground, this field varies sinusoidally with the height of the aipole, so the sinusoidal illumination function is a special case of the complex conjugate excitation function. In general, let $H_{n}(\theta)$ be the radiation field of the nth dipole located at height $h_{n}$. Here $\theta$ is the elevation angle. With an excitation function $W_{n}\left(\epsilon_{0}\right)$ suitable for producing the main beam in the direction $\theta_{0}$, the array radiation pattern becomes

$$
H(\theta)=\sum_{n=0}^{N-1} W_{n}\left(\theta_{0}\right) H_{n}(\theta)
$$

Note that $H_{n}(\theta)$ is complex, and implicitly contains the phase due to the height $h_{n}$ of the dipole above the ground. The convertiona! linear phase excitation function for a phased array is

$$
W_{n}\left(\theta_{0}\right)=\exp \left(-j\left[h_{n} \sin \theta_{0}\right)\right.
$$


which is appropriate for an array of identical radiators in free space. If the dipoles are located over a perfectly conducting ground so that true images are formed, then (Waldman, 1970)

$$
W_{n}\left\{\theta_{0}\right\}=\cos \left(B h_{n} \sin \theta_{0}\right)
$$

which is the sinusoidal excitation function. The complex conjugate excitation function is the choice

$$
W_{n}\left(\theta_{0}\right)=H_{n}^{*}\left(\theta_{0}\right)
$$

\subsection{Effects of Excitation Function}

The array radiation pattern has been studied using these three types of excitation functions and the five different ground conditions mentioned in section 3. The results are shown in Figs. 5 (low-angle steering) and 6 (high-angle steering). For low-angle steering it is seen that the complex conjugate excitation function generally produce; a narrower beam as well as smaller sidelobes than the conventional excitation. Also, the actual direction of the main beam maximum agrees best with the design steering angle for the conjugate excitation (also see Table 1). Sinusoidal excitation proauces distorted beam shapes as well as high sidelobes.

For high-angle steering (Fig. 6), we see that the agreement between main beam direction and steering angle is best for conjugate ana sinusoidal than for the conventional excitations. For all ground systems considerea, this conclusion holds. Also, the beam shapes are better and the beamwioth 
is narrower for the conjugate and sinusoidal excitations, Sinusoidal excitation provices the lowest sidelobe levels for high-angle steering.

it is not possible to effectively steer the beam to elevation angles higher than about $75^{\circ}$ without creating major undesired sidelobes, or lower than about $9^{\circ}$, irrespective of the type of excitation function useo. Finally, conjugate and sinusoidal excitations provide a larger beam steering range than does conventional excitation.

\subsection{Effect of Ground System}

The type of ground system used has an influence on the width, shape, and pointing angle of the main beam at both low and high steering angles. Figure 7 illustrates this effect for the case when the complex conjugate excitation function set for steering angles of $10^{\circ}$ and $70^{\circ}$ is used. Similar figures drawn for other steering angles show that for low-angle steering, lower conductivity earth with or without a ground system produces a narrower beam than higher conductivity earth. Table 1 compares the pointing angles and beamwidths for various ground systems for two types of excitation. The lower limit for beam steering is about $9^{\circ}$ for all the ground systems considered. The presence of a radial wire ground system does not improve the low-angle performance of the array. However, when the array is excited to steer the beam to high elevation angles, the presence of the radial wire ground system markediy reduces the beamwidth. Also it brings the actual main beam closer to the steering angle chosen for the excitation function. A ground system consisting of a large number of radial wires (approaching the perfectly conducting disc ground system) gives the best beam performance 
at high elevation angles. These effects are functions of the radius of ground systems used [Teng, 1978]. Again, the bear cannot be effectively steered beyond $75^{\circ}$ elevation without creating objectional sidelobes, irrespective of the excitation function or the type of ground system used.

\subsection{DISCUSSION}

A study of the radiation pattern of a VED over an inhomogeneous ground system reveals that it is a function of both the height of the VED above the ground and the properties of the ground. The radiated field is in general complex and both the magnitude and phase change with height and ground properties. Thus, the design of a vertical array of VEDs over an inhomogeneous ground system is analogous to the design of an array of dissimilar antennas. Classical excitation to achieve a desired steering angle is not applicable. He have studied the behavior of the array pattern using complex conjugate and sinusoidal excitation functions and have compared the results with those for the conventional phased array excitation. For a variety of ground systers considered, both the complex conjugate and the sinusoidal excitations produce better results at highsteering angles than does convenicional excitation. The performance improvement is with respect to the beam shape, beamwiath, siaelooe level, and agreement between desired and actual directions of the main beam.

It is found that over ground conoitions usually encountered in practice, it is impossible to steer the beam to very low or very high elevation angles. For a 21 element array of height $2 \lambda$, the steering limits are roughly $9^{\circ}$ and $75^{\circ}$. For such an array it is found that for low 
elevation angles the type of ground system used has very little influence on the main beam. However, the performance is better over dry, poorlyconducting earth $\left(\varepsilon_{r}=5, \sigma=10^{-4} \mathrm{~S} / \mathrm{m}\right)$ than a moist, well-conducting earth $\left(\varepsilon_{r}=15, \sigma=10^{-2} \mathrm{~S} / \mathrm{m}\right)$. For high elevation angles a good ground system with a large number of radial wires improves the performance considerably.

The basic constituent of this prefiminary study is the availability of a formulation to evaluate the fields of a VED above an azimuthally symmetric inhomogeneous ground. The complex conjugate excitation function is derived from this formulation. If ground conditions are such that the actual radiation pattern of a VEO for various heignts cannot be evaluated, the complex conjugate excitation function cannot be determined. In such a case, a sinusoidal excitation function appears to be a better choice than the conventional excitation.

The problem of designing an excitation function to synthesize a given beam pattern is, in general, very aifficult. However, it is possible to synthesize a beam with specified nulls and/or specified (complex) radiation field at various elevation angles. The excitation function for achieving such a beam is obtained by solving a set of equations generated from (14), viz.,

$$
H\left(\theta_{i}\right)=\sum_{n=0}^{N-1} W_{n} H_{n}\left(\theta_{i}\right), \quad i=1,2, \ldots, m
$$

where the array pattern $H\left(\theta_{j}\right)$ is specified for m elevation angles. Since $H$, $W_{n}$ and $H_{n}$ are coinplex, one needs $2 N$ constraints on $H(\theta)$ (i.e., $m=2 N$ ) to determine the excitation function uniquely. However, for a given set of constraints, one can determine an excitation function $w_{n}$ which will (a) 
satisfy the constraints if $62 \mathrm{~N}$, and (b) produce a best fit to the specifications if $m>2 N$. But in terms of the general problem of an antenna array design, the specifications are usually in terms of beamwidth, pointing angle, beam shape, sidelobe level, sidelobe ioctions, and null locations. Most of these parameters are explicit and, hence, not easily usable in solving for the excitation function $w_{n}$ from (18). Thus, the design of antenna arrays over inhomogeneous ground systems needs further detailed investigation. 


\section{REFERENCES}

1. Balanis, C. A. and D. DeCarlo (1982), "Monopole antenna patterns on finite size composite ground planes," IEEE Trans. On Antennas and Propagation, AP-30 (4), pp. 764-768.

2. de Jong, G. (1975), "Electromagnetic wave propagation over an inhomogeneous flat earth (two-dimensional integral equation formulation)," Radio Sci. 10(12), pp. 925-933.

3. FitzGerrel, R. G., A. C. Wilson, L. L. Proctor and H. V. Cottony (1967), "An HF array antenná electrunically scanneo in elevation," IEEE Trans. on Antennas and Propagation, AP-15(6), PP. 758-766.

4. Hufford, G. A. (1952), "An integral equation approach to the problem of wave propagation over an irregular terrain," Quart. J. Appl. Math, g(4), pp. $391-404$.

5. King, R. J. (1969), "Electromagnetic wave propagation over a constant impedance plane," Radio Sci., 4(3), pp. 356-374.

6. Linz, P. (1969), "Numerical method for Volterra integral equation with singular kernels," SIAM J. Numerical Analysis, 6(3), pp. 356-374.

7. Mittra, R. (1961), "A vector form of compensation theorem and its application to boundary value problems, "AFCRL 75, University of Colorado, Boulder.

8. Monteath, G. D. (1951), "Application of compensation theorem to certain radiation and propagation problems," Proc. Inst. Elec. Eng., 98, Pt IV, pp. 23-3l.

9. Pierce, W. H. (1957), "Numerical integration over the planar annulus," J. Soc. Indus. Appl. Math., 5(2), pp. 66-73.

10. Rafuse, R. P. and J. Ruze (1975), "Low-angle radiation from vertically polarized antennas over radially heterogeneous flat grouna," Radio Sci., 10(12), pp. 1011-1018.

11. Teng, C. (1978), Ground Wave Propagation and Raoiation Patierns of a Vertical Electric Dipole over a Radially Varying Surface Impedance Plane, Ph. D. Thesis, University of Wisconsin-Madison, Madison, WI.

12. Teng, C. J. and R. J. King (1981), "Surface fielas and radiation patterns of a vertical electric oipole over a radial-wire ground system," Electromagnet ics, 1(1), pp. 101-176.

13. Hait, J. R. (1962), Electromagnet ic Waves in Strat if ied Media, Pergamon Press, NY. 


\section{$-18-$}

14. Wait, J. R. and $K$. Spies (1970), "On the radiation from a vertical dipole
with an inductive wire-grid ground system," IEEE Trans. On Antennas and
Propagation, AP- $18(4)$, pp. 558-560.

15. Waldman, A. (1970), "Elevation steering of the pattern of a vertical
array of vertically polarized elements over a ground screen " IEE
on Antennas and Propation, Ap on Antennas and Propagation, AP-18(1), pD. 105-107.

\section{DISTCLALMER}

This doxwmed was prepared as an account of work sponsored by an ageicy of the I nited States Government. Neither the Linited States Goverment nor the I niversity of Califomia nur any of their employees, makss any watranty, express or implied, or asedmes any legal liability of resporsibility for the accuracy, completeness, ac usefutness of any iaformation. apparatus, product, or process dicclosed, or represems sthat its use nould not infringe privalely owned rights. Reference herpin to any specilsc commercial products, process. of service by srade name. tradenark. manufacturer, or otherwise, does not naressarily constitute or imply is endorsentent, recommendation. of fasoring us the l'nited States cionemment or the laiversity of Caljfornia. The siews and oplnions of authors axpressed herein do not necessarily state or leflect hose of the inited States Gavemment thereof and shall not be used for adverlising or product endorsement purposes. 
Table 1

Effect of Ground System and Excitation Function on Low-angle Steering

\begin{tabular}{|c|c|c|c|c|c|c|c|c|c|c|c|c|c|c|c|c|c|c|c|c|}
\hline \multirow{3}{*}{$\begin{array}{l}\text { Design } \\
\text { Steering } \\
\text { Angle } \\
\text { (Oeg) }\end{array}$} & \multicolumn{10}{|c|}{ g Angle (Deg) } & \multicolumn{10}{|c|}{ Half-Power Beamwidth (Deg) } \\
\hline & \multicolumn{2}{|c|}{1} & \multicolumn{2}{|c|}{2} & \multicolumn{2}{|c|}{3} & \multicolumn{2}{|c|}{4} & \multicolumn{2}{|c|}{5} & \multicolumn{2}{|c|}{1} & \multicolumn{2}{|c|}{2} & \multicolumn{2}{|c|}{3} & \multicolumn{2}{|c|}{4} & \multicolumn{2}{|c|}{5} \\
\hline & a & $\mathbf{b}$ & a & b & a & b & a & $t$ & & b & a & $\mathbf{b}$ & a & b & a & $\mathbf{b}$ & a & b & a & $\mathbf{b}$ \\
\hline 0 & 8 & 9 & 9 & 8 & 9 & 8 & 9 & $\varepsilon$ & 0 & 0 & 11.2 & 16.0 & 10.5 & 10.5 & 10.8 & 10.8 & 10.9 & 10.5 & -- & -- \\
\hline 2 & 8 & 9 & 9 & 8 & 9 & & 9 & 8 & 0 & $\mathbf{0}$ & 12.6 & 16.0 & 10.9 & 10.7 & 11.2 & 10.8 & 11.1 & 10.5 & -- & -- \\
\hline 4 & 8 & $\mathbf{g}$ & 9 & 8 & 9 & 8 & 9 & $\varepsilon$ & 0 & 0 & 14.0 & 16.2 & 11.5 & 10.8 & 12.0 & 11.0 & 11.8 & 10.7 & -- & -- \\
\hline 6 & & 10 & 9 & 9 & 10 & 9 & 9 & 8 & 0 & 0 & 15.0 & 17.0 & 12.0 & 11.2 & 12.9 & 11.2 & 12.6 & 11.0 & -- & -- \\
\hline 8 & 10 & 11 & 10 & 9 & 10 & 9 & 10 & 9 & 0 & 0 & 16.5 & 17.5 & 13.0 & 11.2 & 14.0 & 11.8 & 13.8 & 11.4 & -- & - \\
\hline 10 & 11 & 12 & 10 & 9 & 10 & 9 & 10 & 9 & 0 & 9 & 17.5 & 17.5 & 14.2 & 12.0 & 15.8 & 12.7 & 14.5 & 12.0 & -- & -- \\
\hline 12 & 12 & 14 & 10 & 10 & 11 & 10 & 10 & 9 & 0 & 14 & 18.3 & 18.4 & 16.4 & 14.0 & 17.5 & 14.7 & 17.9 & 13.8 & -- & 18.6 \\
\hline 14 & 14 & 15 & 11 & 11 & 12 & 11 & 11 & 10 & 0 & 16 & 18.9 & 18.1 & 18.5 & 16.8 & 19.5 & 17.0 & 20.2 & 16.8 & -- & 12.2 \\
\hline 16 & 15 & 16 & 13 & 13 & 15 & 14 & 13 & 12 & 17 & 17 & 19.3 & 18.7 & 21.0 & 19.8 & 21.2 & 19.2 & 22.8 & 21.0 & -- & 12.0 \\
\hline 18 & 16 & 17 & 17 & 18 & 18 & 18 & 18 & 17 & 17 & 17 & 20.2 & 19.0 & 22.5 & 21.0 & 22.0 & 19.8 & 24.0 & 23.2 & 16.4 & 12.1 \\
\hline 20 & 17 & 19 & 19 & 20 & 20 & 20 & 20 & 20 & 17 & 18 & 21.5 & 19.5 & 23.0 & 20.5 & 22.0 & 18.5 & 25.0 & 24.7 & 16.0 & 12.9 \\
\hline
\end{tabular}

Ground Systems: (1) $\varepsilon_{r}=15, \sigma=10^{-2}$ with radial wires, (2) $E_{r}=5, \quad \sigma=10^{-4}$ with radial wires

(3) $\epsilon_{r}=5, \quad \sigma=10^{-4}$ with disc, (4) $\varepsilon_{r}=5, \quad \sigma=10^{-4}$ homogeneous ground,

(5) perfectiy conducting homogeneous ground.

Excitatiois: (a) Conventional, (b) Complex Conjugate 


\section{Figure Captions}

Figure 1. Geometry of a VED over an azimuthally symmetric ground having surface impedance $Z_{s}^{\prime}(\rho)=n_{0} \Delta^{\prime}(\rho)$. The HMD is a test source for measuring $H_{\phi}$.

Figure 2. Magnitude and Phase of $\Delta^{\prime}(\rho)$ for a radial wire ground system $(N=120)$ an well- and poorly-conducting earth. $f=10 \mathrm{MHz}$.

Figure 3. Radiation pattern amplitude of a VED over various ground systems as a function of height, $h_{1} . f=10 \mathrm{MHz}$.

Figure 4. Radiation pattern phase of a VED over various ground systems as a function of height, $h_{1} . f=i 0 \mathrm{MHz}$.

Figure 5. Low-angle steered radiation patterns for a stacked array of VEDs centered on a radial wire ground system $(N=120, b=2 \lambda)$ lying on poor earth $\left(\varepsilon_{r}=5, \sigma=10^{-4} \mathrm{~S} / \mathrm{m}\right) . \quad f=10 \mathrm{MHz}$.
Excitations: (a) conventional
(b) complex conjugate
(c) sinusoidal 


$$
-21-
$$

Figure 6. Steered radiation patterns for a stacked array of VEDs centereo on a radial wire ground system $(N=120, b=2 \lambda)$ lying on poor $\operatorname{earth}\left(\varepsilon_{r}=5, \sigma=10^{-4} \mathrm{~s} / \mathrm{m}\right) . f=10 \mathrm{MHz}$.

\author{
Excitations: (a) conventional \\ (b) complex conjugate \\ (c) sinusoidal
}

Figure 7. Low-angle $\left(10^{\circ}\right)$ and high-angle $\left(70^{\circ}\right)$ steered radiation patierns for a stacked array of VEus on different grounos, using a conjugate excitation function. $f=10 \mathrm{MHz}$. 


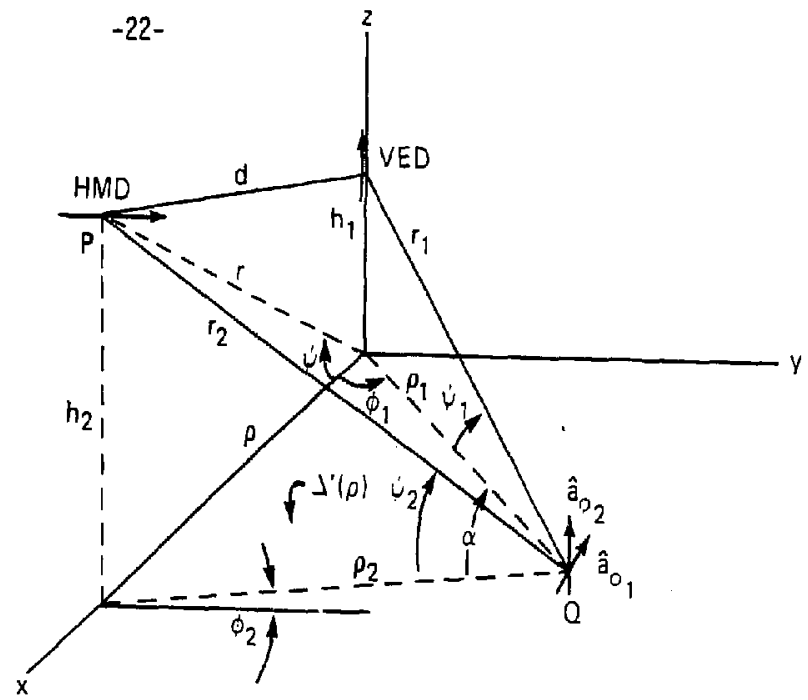

Figure 1. Geometry of a VED over an azimuthally symmetric ground having surface impedance $Z_{S}^{\prime}(p)=\eta_{0} \Delta^{\prime}(\rho)$. The HMD is a test source for measuring $H_{\phi_{\phi}^{\prime}}^{\prime}$.

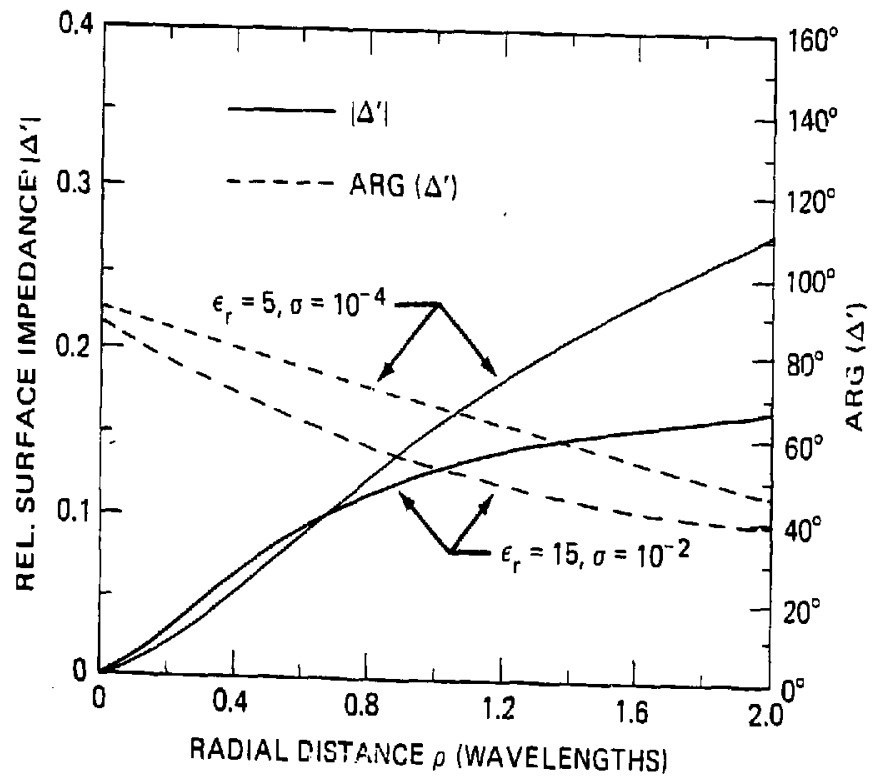

Figure 2. Magnitude and Phase of $\Delta^{\prime}(\rho)$ for a radial wire ground system $(N=120)$ on well- and poorly-conducting earth. $f=10 \mathrm{MHz}$. 


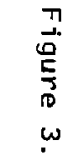

FIELD AMPLITUDE

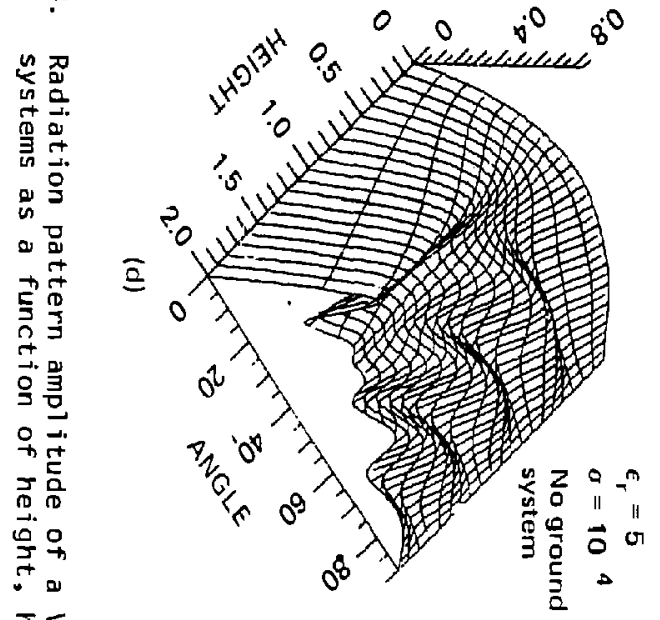

于.5

)

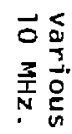

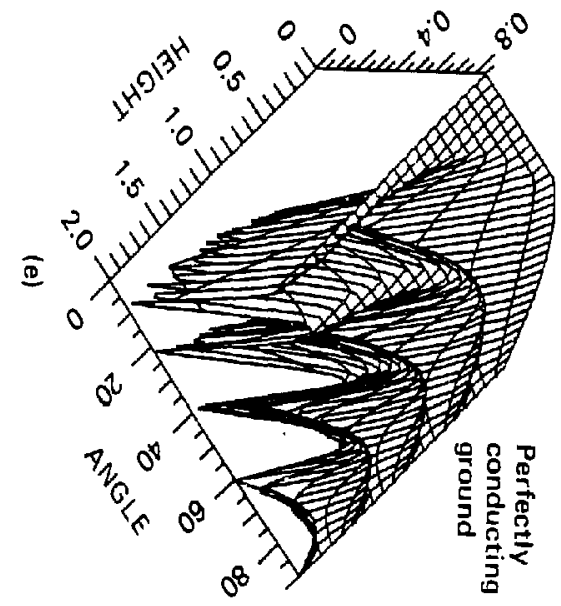

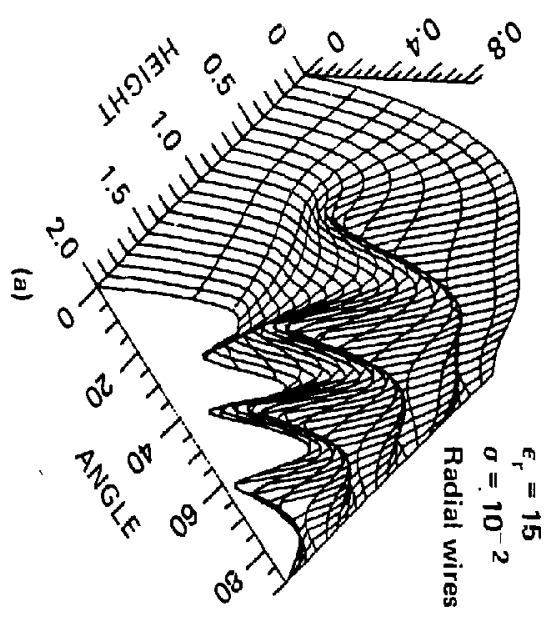

FIELD AMPPLITUDE

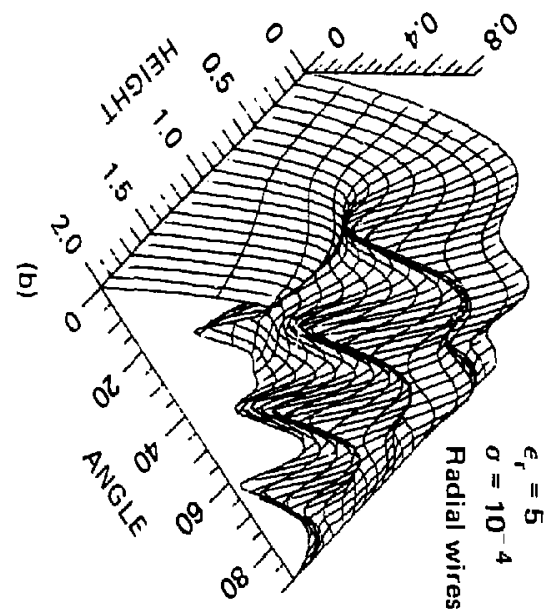

FIELD AMPLITUDE

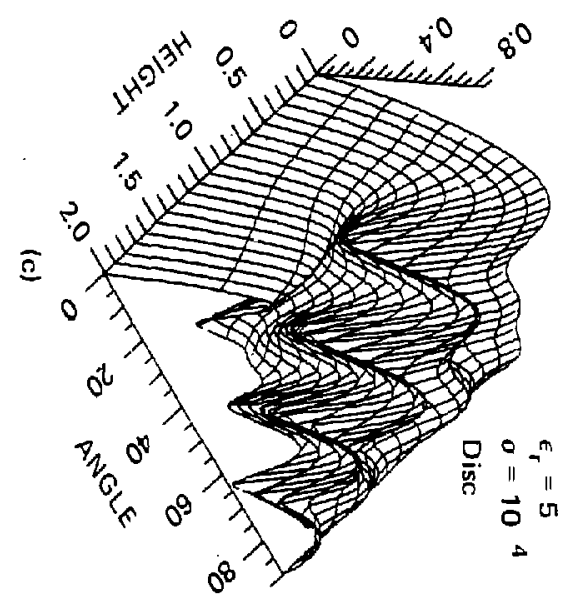




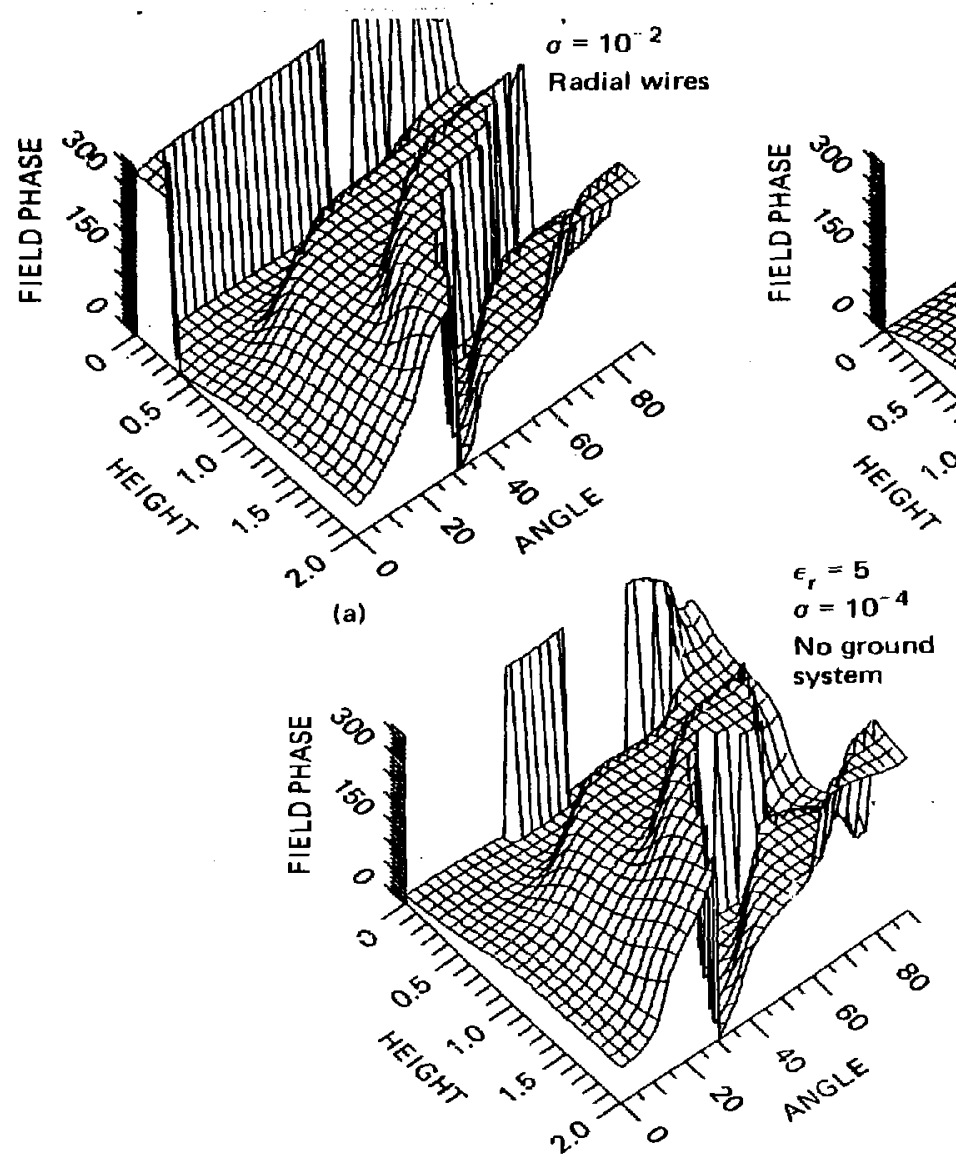

(d)

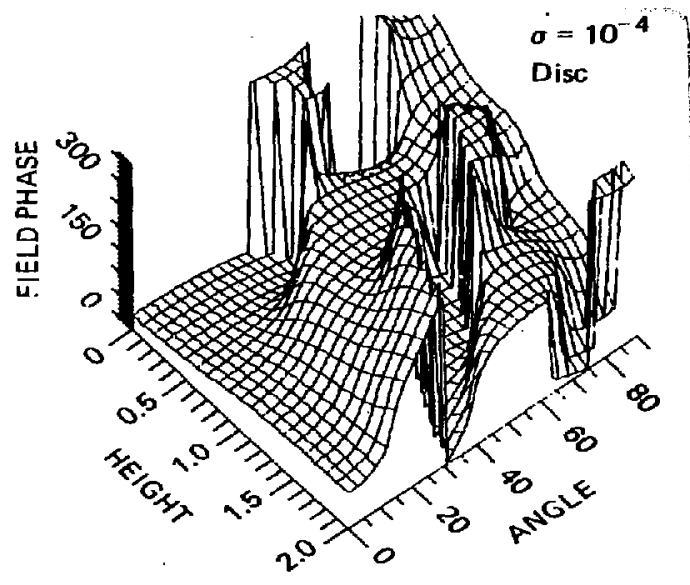

(c)

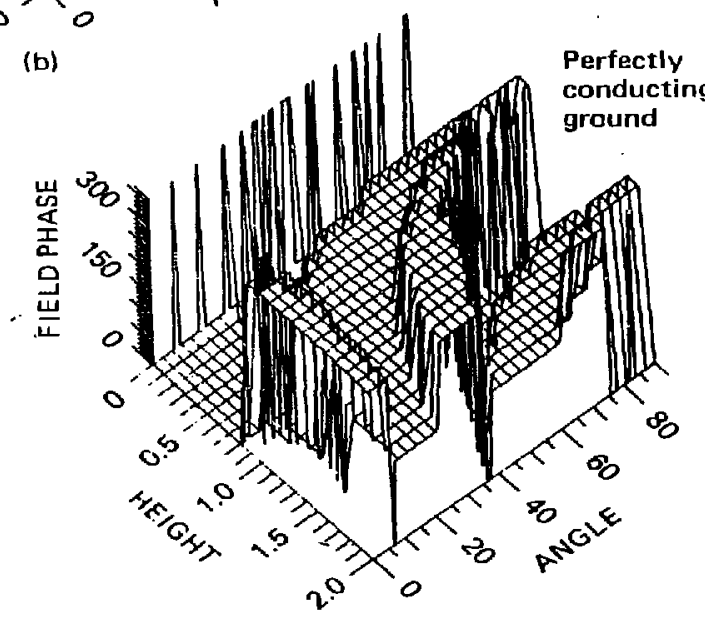

(e)

Figure 4. Radiation pattern phase of a VED over various ground systems as a function of height, $h_{1} . f=10 \mathrm{MHz}$. 


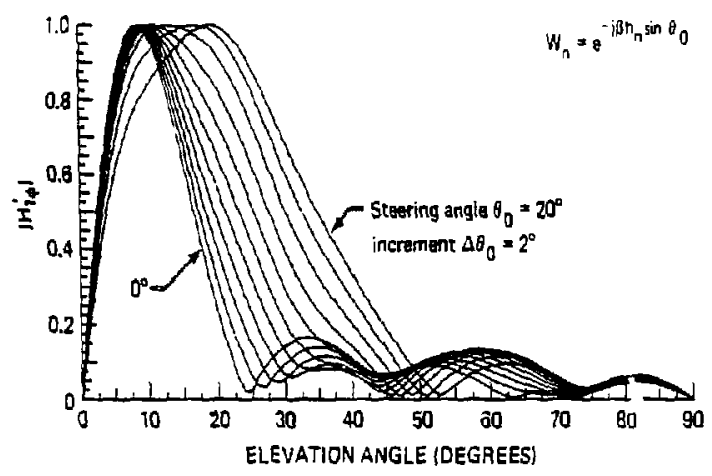

(a)

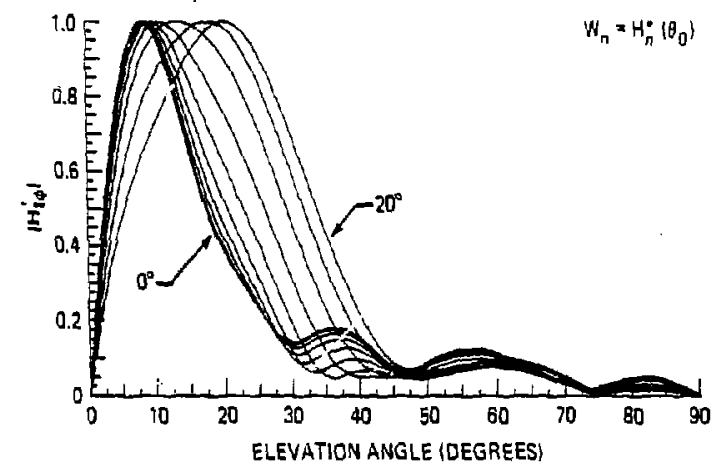

(b)

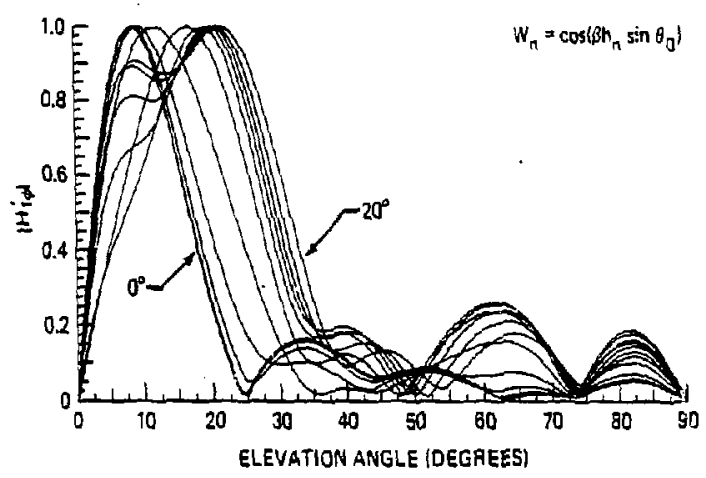

(c)

Figure 5. Low-angle steered radiation patterns for a stacked array of VEDs centered on a radial wire ground system $(N=120, b=2 x)$ lying on poor earth $\left(\varepsilon_{r}=5, \sigma=10^{-4} \mathrm{~S} / \mathrm{m}\right), \quad \epsilon=10 \mathrm{MHz}$.

Excitations: (a) conventional (b) complex conjugate (c) sinusoidal 


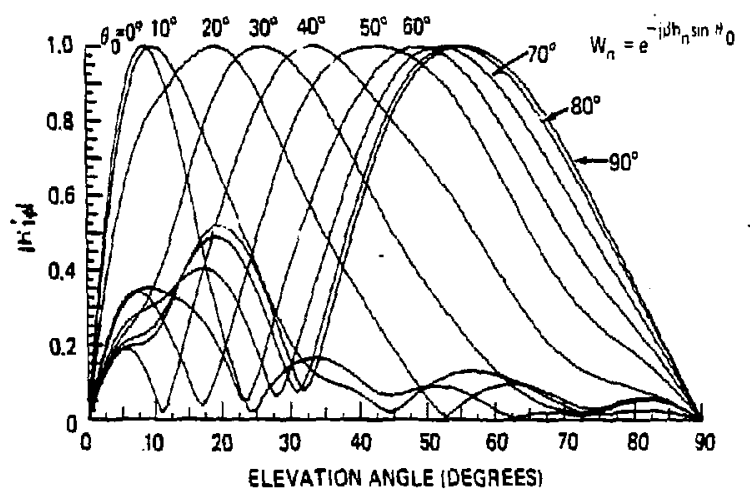

(a)

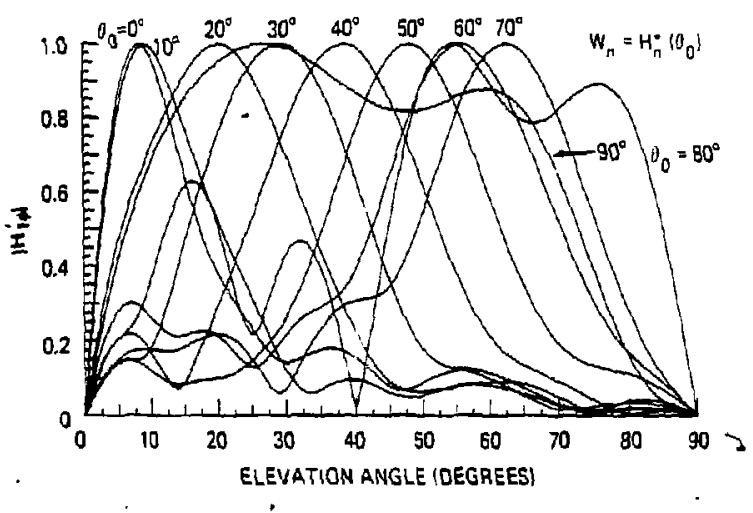

(b)

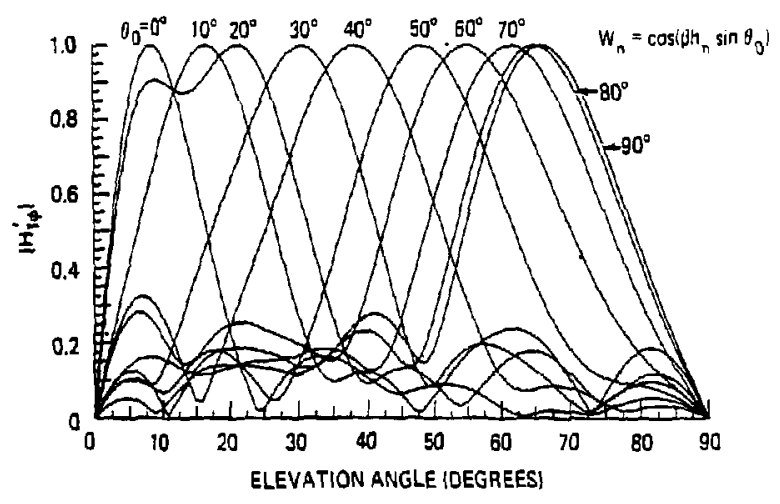

(c)

Figure 6. Steered radiation patterns for a stacked array of VEDS centered on a radial wire ground system $(N=120, b=2 \lambda)$ lying on poor earth $\left(\varepsilon_{r}=5, \sigma=10^{-4} \mathrm{~s} / \mathrm{m}\right) . \quad f=10 \mathrm{MHz}$.

Excitations: (a) conventional (b) cnmplex conjugate (c) sinusoida] 


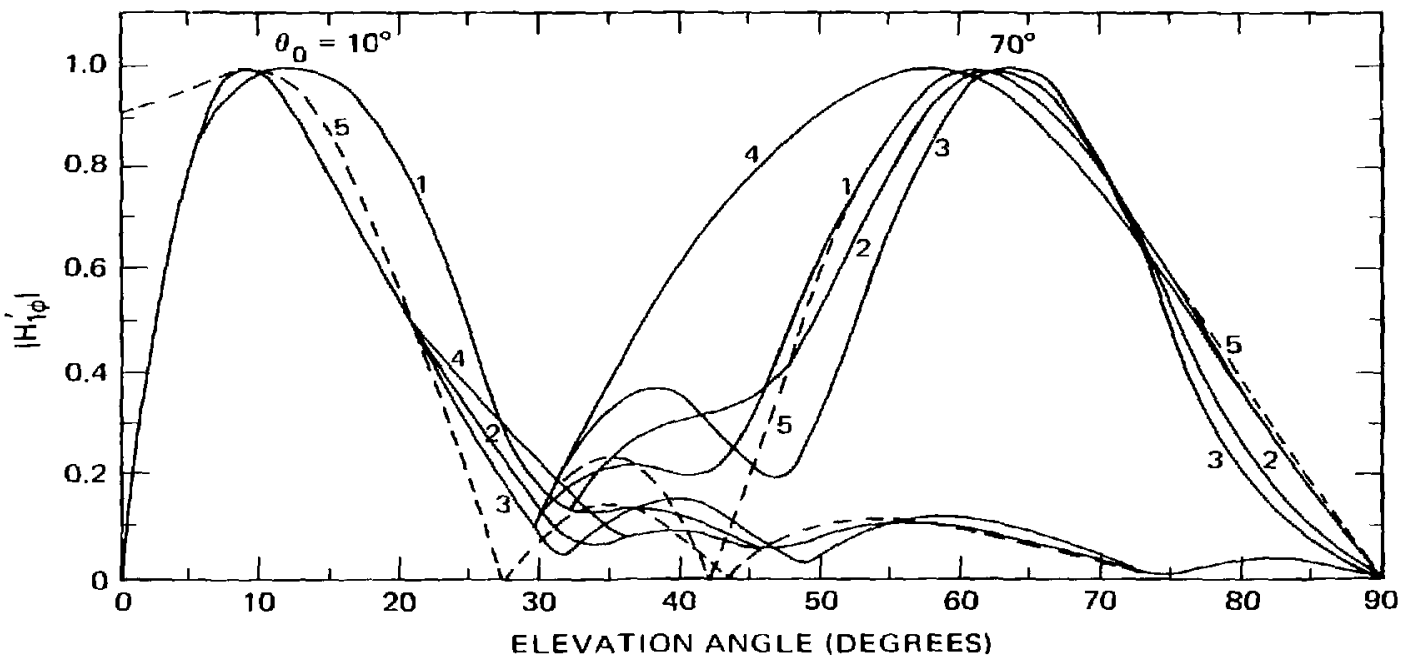
$1 \epsilon_{\mathrm{r}}=15, \sigma=10^{-2}$, radial wires
$2 \epsilon_{r}=5, \sigma=10^{4}$, radial wires
$3 \varepsilon_{\mathrm{r}}=5, a=10^{-4}$, disc
$4 \varepsilon_{r}=5, a=10^{-4}$, no ground system
5 Perfectly conducting earth

Figure 7. Low-angle $\left(10^{\circ}\right)$ and high-angle $\left(70^{\circ}\right)$ steered radiation patterns for a stacked array of VEDs on different grounds, using a conjugate excitation function. $f=10 \mathrm{MHz}$ 